\title{
Communication \\ Study of Very Forward Neutrons with the CMS Zero Degree Calorimeter
}

\author{
Olivér Surányi on Behalf of the CMS Collaboration \\ MTA-ELTE Lendület CMS Particle and Nuclear Physics Group, Eötvös Loránd University, 1053 Budapest, \\ Hungary; oliver.suranyi@cern.ch
}

Received: 15 August 2019; Accepted: 5 October 2019; Published: 9 October 2019

\begin{abstract}
Forward neutrons are studied in proton-lead collisions at the CMS experiment at the CERN LHC. They provide information on the centrality and event plane of collisions and provide an opportunity to study nuclear breakup. At the CMS experiment they are detected by the Zero Degree Calorimeters (ZDCs) in the $|\eta|>8.5$ pseudorapidity range. The ZDCs are quartz fiber Cherenkov calorimeters using tungsten as absorber. Test beam data and events with a single spectator neutron are used for the calibration of these detectors. A Fourier-based method is used correct for the effect of multiple $\mathrm{pPb}$ collisions. The corrected ZDC energy distribution is used to calculate centrality percentiles and unfold the neutron multiplicity distribution.
\end{abstract}

Keywords: forward neutrons; zero degree calorimetry; centrality; heavy ions

\section{Introduction}

Very forward $(|\eta|>8.5)$ neutrons are produced in hadron-nucleus and heavy ion collisions. The main physics processes involved are intranuclear cascades [1], nuclear evaporation [2], and nuclear resonances, like the giant dipole resonance [3]. The information gathered from the observation of these neutrons can be used to tag ultraperipheral collisions, calculate the event plane and estimate centrality in heavy ion and hadron-nucleus collisions. Cascade and evaporation nucleons were observed and studied by a wide range of fix target experiments [4]. Ultraperipheral collisions accompanied by nuclear resonances were studied by the STAR Collaboration at RHIC [5,6]. In the ALICE experiments at LHC cascade and evaporation neutrons are used for the estimation of centrality $[7,8]$. The energy spectrum of very forward neutrons produced in proton-proton collisions was measured by the LHCf experiment $[9,10]$. In the CMS experiment these neutrons can be observed by the Zero Degree Calorimeters (ZDC). The most important models, which include forward neutron production are DPMJet [2], URQMD [11], Geant4 [12,13], SMASH [14], JAM [15], and PHSD [16].

\section{The Zero Degree Calorimeter of CMS Experiment}

The two ZDCs are located in the neutral particle absorber (TAN), roughly $\pm 140 \mathrm{~m}$ away from the CMS interaction point, between the two beampipes. They measure neutral particles at pseudorapidity values $|\eta|>8.5$, as the charged products are removed by dipole magnets located between the central CMS detectors and the ZDCs. They are Cherenkov sampling calorimeters consisted of cladded quartz fibers and tungsten plates. They have three different sections (Figure 1): the electromagnetic (EM) section, the hadron (HAD) section, and the reaction plane detector (RPD). The EM section is 19 radiation lengths long, which is equal to one interaction length and has five transverse segments. The RPD is a $4 \times 4$ array of quartz tiles and is used to determine the event plane for flow measurements in heavy ion collisions. The HAD section is 5.6 hadronic interaction length and has four longitudinal segments. The plates in the HAD section are tilted by $45^{\circ}$ to maximize the light yield of the fibers. This paper presents results using the EM and HAD sections of the ZDC detector on the lead going side $(z<0)$ 
measured in $\mathrm{pPb}$ collisions at the CMS experiment in 2016. A more detailed description of the ZDC detectors can be found in references [17-20].

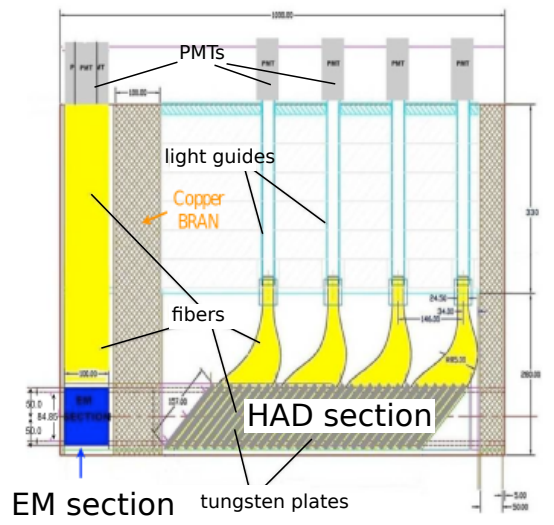

\section{ZDC Layout}

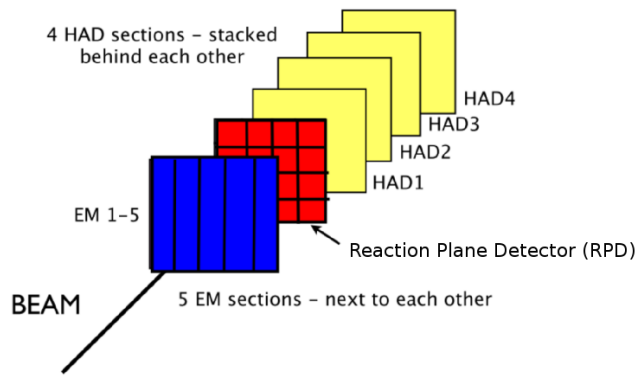

Figure 1. The side view of the CMS Zero Degree Calorimeter (left) and the segmentation of each sections (right) [21].

Every channel of the detector is read out in 10 timeslices (denoted with TS0 - TS9), each 25 ns long. The main signal arrives in TS4 as shown in the left panel of Figure 2. The bunch spacing in the $2016 \mathrm{pPb}$ datataking was $100 \mathrm{~ns}$, so additional collisions may happen four timeslice before or after the primary signal (out-of-time pileup). The signal value $Q_{i}$ for a certain $i$ channel is extracted as:

$$
Q_{i}=Q_{i, \mathrm{TS} 3}-\frac{1}{2}\left(Q_{i, \mathrm{TS} 2}+Q_{i, \mathrm{TS} 6}\right),
$$

where $Q_{i, \text { TSX }}$ is the signal value in the $X$ th timeslice for channel $i$. The second term is used to subtract contribution from pedestal and the tail of out-of-time pileup events.
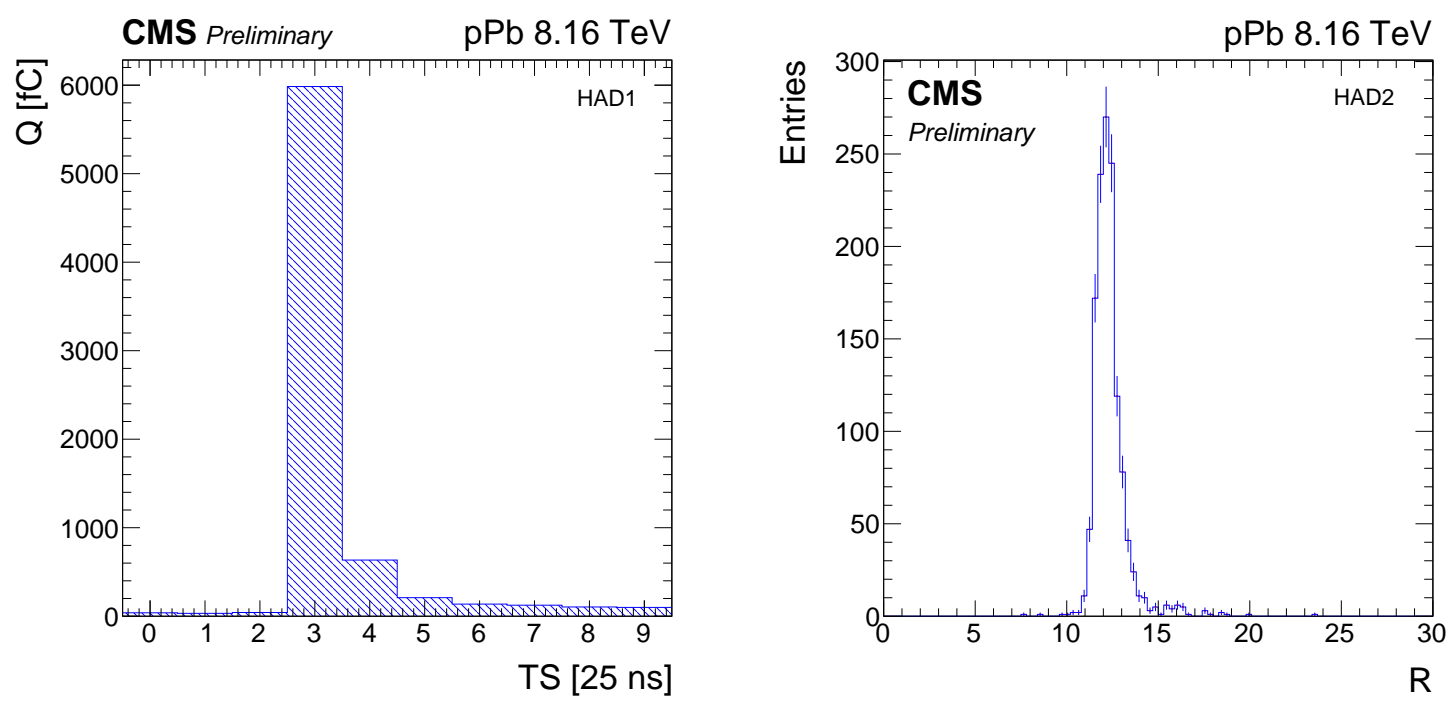

Figure 2. The time dependence of the Zero Degree Calorimeters (ZDC) signal (left) and the distribution of TS4 and TS5 signal ratio (right) [21].

In order to increase the dynamic range of the detector, the largest signals are let to slightly saturate and their signal values are calculated by scaling up the sum of TS4 and TS5 by a scale-factor $\langle R\rangle$. The scale factor is calculated separately for each channel from the distribution of the values: 


$$
R=\frac{Q_{i, \mathrm{TS} 3}-\frac{1}{2}\left(Q_{i, \mathrm{TS} 2}+Q_{i, \mathrm{TS} 6}\right)}{Q_{i, \mathrm{TS} 4}-\frac{1}{2}\left(Q_{i, \mathrm{TS} 2}+Q_{i, \mathrm{TS} 6}\right)}
$$

which is shown in the right panel of Figure 2. The value of $\langle R\rangle$ is calculated from the mean of this distribution. This method ensures that both the resolution remains good for the low-energy signals and the whole energy range of the detector can be used.

\section{Calibration}

The relative gains of the different channels are matched (intercalibrated) by using distributions of signal ratios between different channels. These are compared to the same distributions from the 2010 $\mathrm{PbPb}$ data collection period, following the test beam calibration of ZDCs. An example of two such distributions is shown in Figure 3.
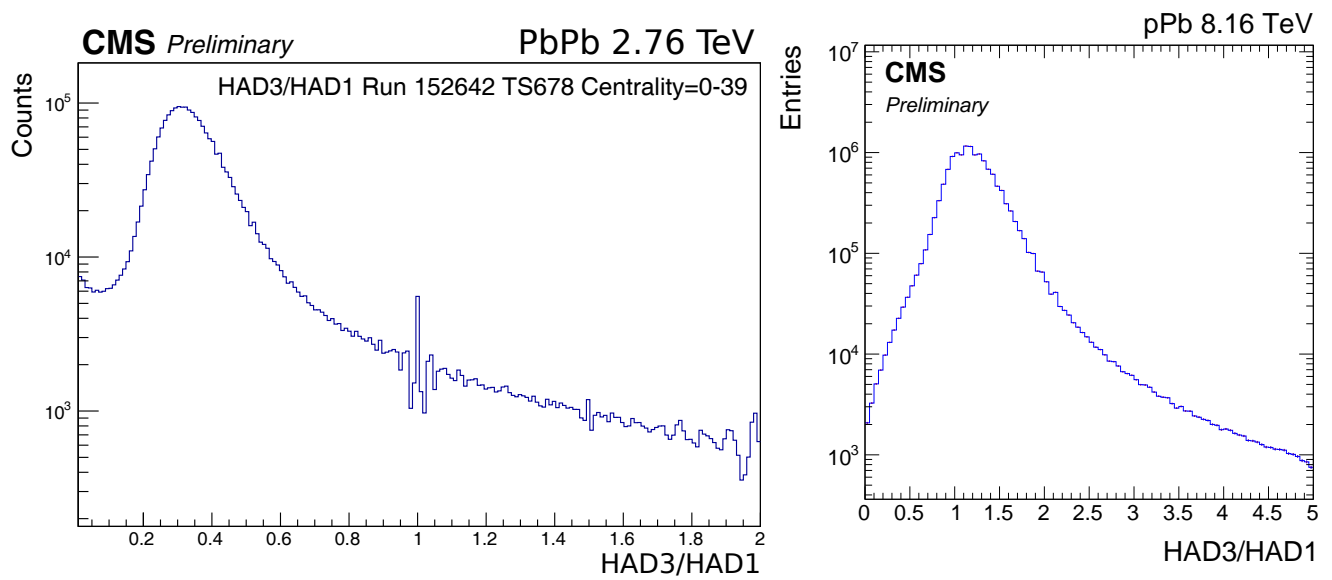

Figure 3. Comparison of signal ratio distributions between $2.76 \mathrm{TeV} \mathrm{PbPb}(\mathbf{l e f t})$ and $8.16 \mathrm{TeV} \mathrm{pPb}$ collisions (right) [21].

The low energy part of the total ZDC signal distribution is shown in Figure 4. Since the slow neutrons are close to being monoenergetic, the peaks correspond to events with single, double, and triple neutrons. The widths of the peaks are due to the slight variation in the neutron energy and the finite resolution of the detector. The spectrum is fitted with the sum of Gaussian shapes, with the n-neutron peak position $\mu_{n}$ and width $\sigma_{n}$ constrained as:

$$
\begin{aligned}
\mu_{n} & =n \mu_{1}, \\
\sigma_{n}^{2} & =n \sigma_{1}^{2},
\end{aligned}
$$

where $\mu_{1}$ and $\sigma_{1}$ are the position and width of the single neutron peak respectively. The results from the fit are used for the absolute energy calibration of the detector, since the position of the first peak corresponds to $2.56 \mathrm{TeV}$, the energy of nucleons in the $\mathrm{Pb}$ ion in $\mathrm{pPb}$ collisions with $8.16 \mathrm{TeV}$ collision energy [22]. The detector resolution is found to be $24 \%$, which is slightly larger than the $15 \%$ resolution extrapolated from the test beam measurement [20]. 


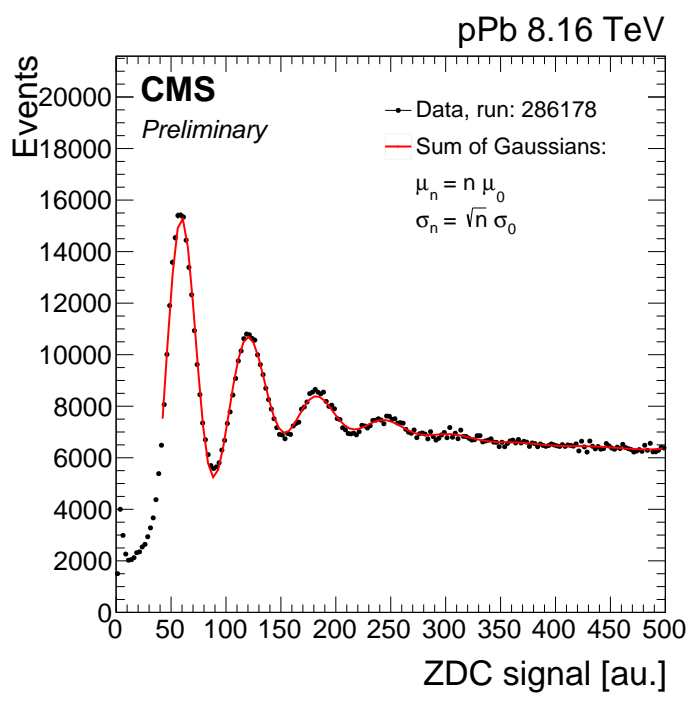

Figure 4. The low end of ZDC signal distribution. The peaks correspond to events with one, two and three neutrons detected by the ZDC respectively. The position of the first peak corresponds to $2.56 \mathrm{TeV}$, the energy of nucleons in the $\mathrm{Pb}$ ion for $8.16 \mathrm{TeV} \mathrm{pPb}$ collisions [21].

\section{Pileup Correction}

The ZDC energy spectrum is shown in Figure 5 for two different data collection periods with different average number of collisions in a single event (pileup). An important feature of these spectra is a shoulder at high energies, which is larger in the data set with a larger pileup. It is caused by pileup collisions. This effect is corrected by the following mathematical procedure, based on the compound Poisson distribution.

Assuming that $n$ is the number of simultaneous $\mathrm{pPb}$ collisions in a single event and it follows a Poisson distribution:

$$
p_{n}=\frac{\mu^{n}}{n !} \frac{\mathrm{e}^{-\mu}}{1-\mathrm{e}^{-\mu}}
$$

where only the $n>0$ case is considered, therefore $1-\mathrm{e}^{-\mu}$ is included in the denominator to ensure the normalization of the above expression. $\mu$ is the average number of collisions in an event, that provide at least one neutron in the ZDC. The ZDC energy deposit is described by a random variable $X$ :

$$
X=\sum_{i=1}^{n} Y_{i}
$$

where $Y_{i}$ is the random variable describing ZDC energy deposit for an event with single collision. The probability density functions of $Y_{i}$ and $X$ are denoted by $g(x)$ and $f(x)$ respectively. The $f(x)$ function can be expressed as:

$$
f(x)=g(x) p_{1}+(g * g)(x) p_{2}+(g * g * g)(x) p_{3}+\ldots,
$$

where the $*$ operation stands for convolution. Then using Fourier transform and the convolution theorem one may write:

$$
\mathcal{F} f=(\mathcal{F} g) p_{1}+(\mathcal{F} g)^{2} p_{2}+(\mathcal{F} g)^{3} p_{3}+\ldots
$$



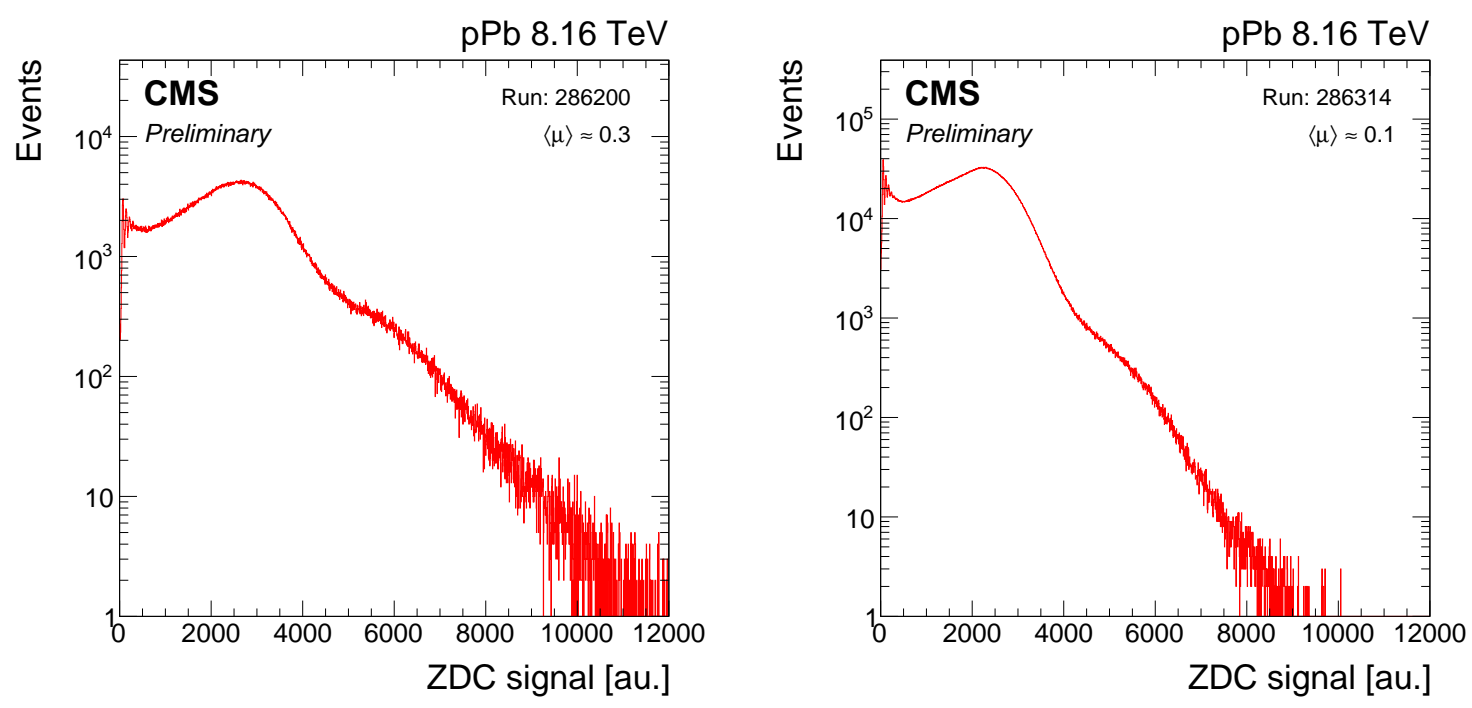

Figure 5. ZDC energy distribution in data sets with average number of collisions 0.3 (left) and 0.1 (right) [21].

After substituting the definition of $p_{n}$ into the equation above, the infinite series becomes a geometrical series and can be summed up to:

$$
\mathcal{F} f=\frac{\mathrm{e}^{-\mu}}{1-\mathrm{e}^{-\mu}}\left(\mathrm{e}^{\mu \mathcal{F} g}-1\right) .
$$

By rearranging this equation, it is possible to express $g(x)$ as:

$$
g(x)=\mathcal{F}^{-1}\left[\frac{1}{\mu} \log \left[\left(\mathrm{e}^{\mu}-1\right) \mathcal{F} f+1\right]\right],
$$

providing a formula to calculate the pileup corrected distribution.

The result of this calculation is shown at three different $\mu$ values in Figure 6. As it is expected the shoulder at high energies disappeared. The method gives a similar result when the value of $\mu$ is varied; there is only a moderate variation in the tail of the distributions.

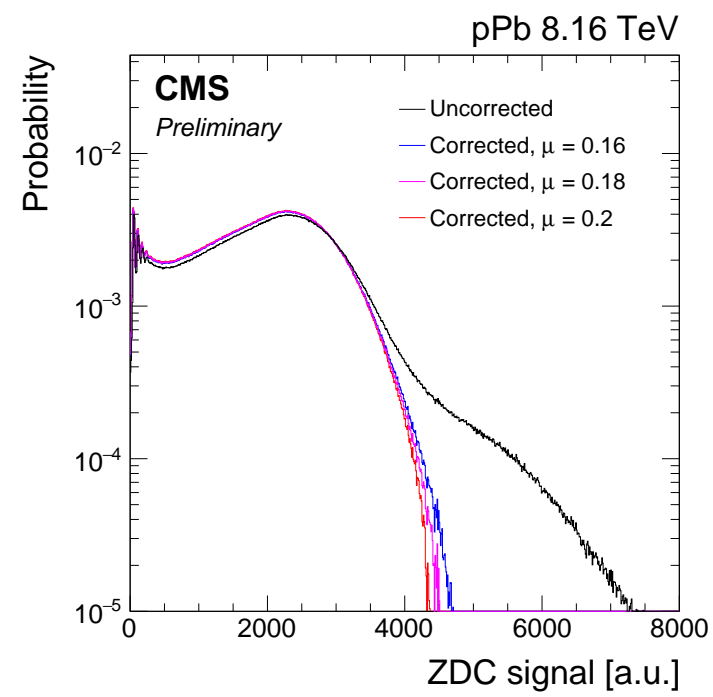

Figure 6. Pileup correction results with Fourier deconvolution method with different $\mu$ values assumed (left) and the percentiles of the ZDC signal distribution, used for centrality determination (right) [21]. 


\section{Using ZDC as a Centrality Estimator in $\mathrm{pPb}$ Collisions}

The typical quantities used to estimate centrality in heavy ion collisions, such as the multiplicity of charged particles, cannot be used in hadron-nucleus collisions, since they are only loosely correlated with the quantities $N_{\text {coll }}$ and $N_{\text {part }}$. A good alternative is to use the very forward energy to estimate centrality, since it is correlated with the number of spectator nucleons. The centrality percentiles, calculated from the corrected energy distribution, are shown in Figure 7. For a proper usage of this zero degree energy as a centrality estimator in a physics measurement, a model of spectator neutron production is needed, which connects the zero degree energy with $N_{\text {coll }}$ and $N_{\text {part }}$. The current models are not valid in the LHC collision energies [1,2,4,12], therefore results from ZDC detectors give a useful input for the development of these models.

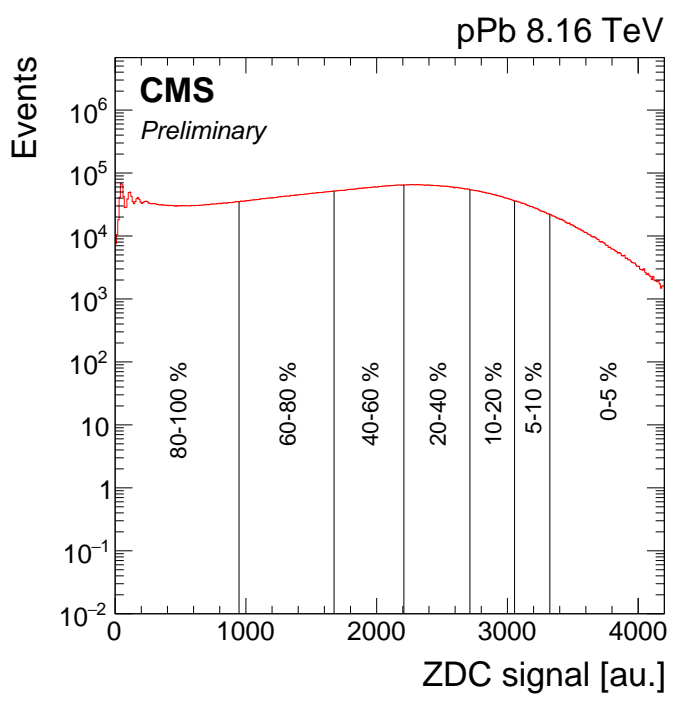

Figure 7. Centrality classes based on ZDC energy [21].

\section{Unfolding Neutron Multiplicity Distribution}

From the fits in Section 3, neutron multiplicity distribution can be calculated for events where only a few neutrons are produced. But the fit cannot be extended to the larger ZDC energy range, as the Gaussian distributions would overlap causing rapid oscillation of the amplitudes. This problem can be circumvented by constraining the amplitude values to change smoothly with the neutron number. This property is achieved by considering the calculation as an unfolding problem. The ZDC response is calculated for $\mathrm{N}$-neutron events and a response matrix $\mathbf{R}$ is constructed (left panel of Figure 8), assuming the linearity of the detector. Using the response matrix, the original fit can be performed by minimalizing a $\chi^{2}$ term. The smoothness of the neutron number distribution is ensured by a linear regularization term (Tikhonov regularization [23]), which requires the first derivative of the distribution to be small. The full $\chi^{2}$ term to minimalize is:

$$
\chi^{2}=(\mathbf{R} \cdot \mathbf{n}-\mathbf{e})^{\top} \mathbf{V}^{-1}(\mathbf{R} \cdot \mathbf{n}-\mathbf{e})+\lambda(\mathbf{D} \cdot \mathbf{n})^{2},
$$

where $\mathbf{n}$ and $\mathbf{e}$ are vectors, whose elements represent the unknown neutron distribution and the measured ZDC spectrum respectively, $\mathbf{V}$ is the covariance matrix of $\mathbf{e}, \mathbf{D}$ is the first derivative matrix and $\lambda$ is the regularization strength.

The optimal solution is calculated by taking the derivative of the above equation, with respect to $\mathbf{n}$. The derivative can be rearranged into the form of linear system of equations:

$$
\left(\mathbf{R}^{\top} \mathbf{V}^{-1} \mathbf{R}+\lambda \mathbf{D}^{\top} \mathbf{D}\right) \mathbf{n}=\mathbf{R}^{\top} \mathbf{V}^{-1} \mathbf{e} .
$$


The optimal $\mathbf{n}$ is calculated from this expression using the LU decomposition method.

The result of this calculation is shown in the right panel of Figure 8. This result serves as a strong constraint and may challenge the models of hadron-nucleus collisions and spectator neutron production [2,11-16].
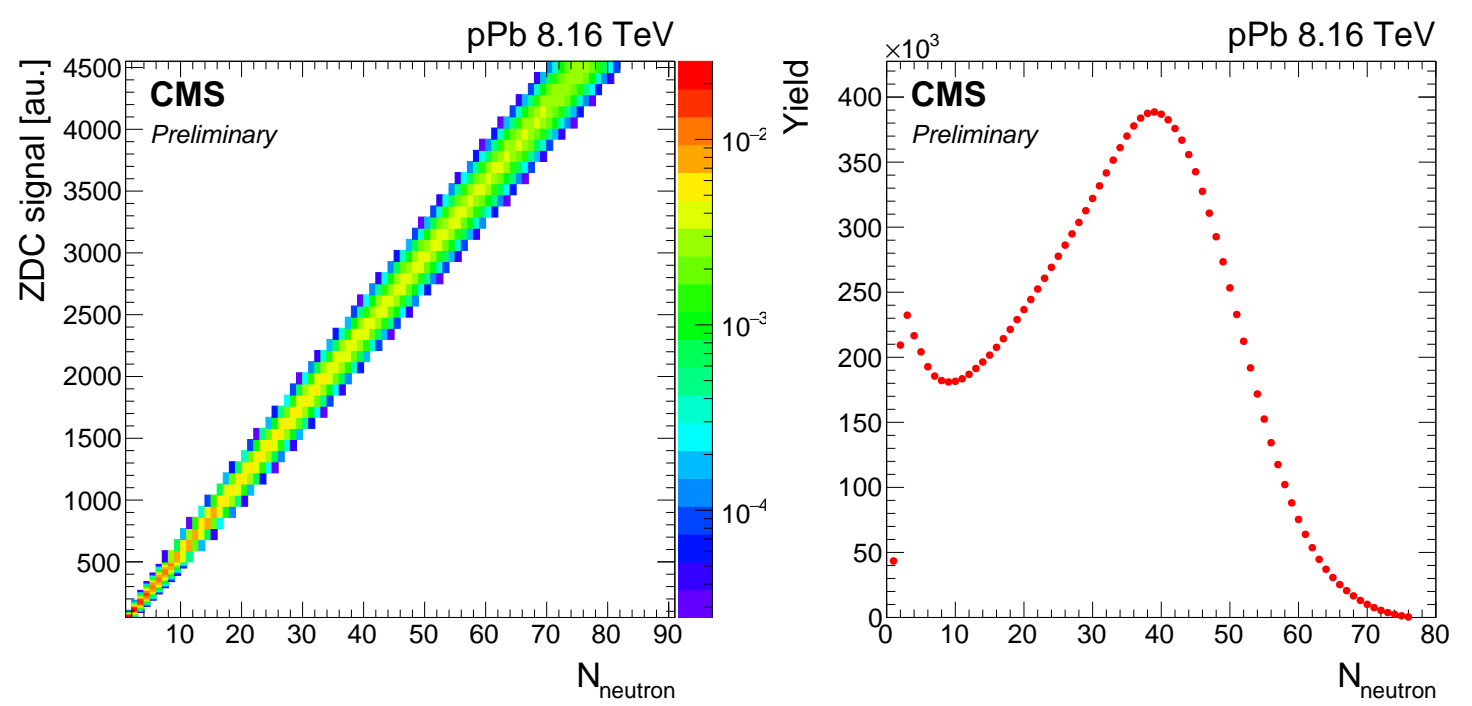

Figure 8. Response matrix (left) and the unfolded neutron multiplicity distribution (right). [21]

\section{Conclusions}

Very forward neutrons are produced in hadron-nucleus and heavy ion collisions via intranuclear cascades, evaporation and nuclear resonances. In the CMS experiment they are observed by the ZDCs. The individual channels of ZDCs in 2016 were gain matched using data collected in 2010. Peaks are observed in the ZDC energy spectrum, which correspond to events with one, two and three nearly monoenergetic spectator neutrons. This provides an opportunity to calibrate the detector and measure the resolution, which is $24 \%$. The pileup effects are corrected using a Fourier deconvolution method. As the very forward energy is suitable to be a centrality estimator, the corrected ZDC energy distribution is used to calculate centrality percentiles. The neutron multiplicity distribution can be unfolded using Tikhonov regularization and the results serve as a constraint for the theoretical nuclear break-up models.

Funding: The CMS Zero Degree Calorimeter detector is supported by the Office of Science, US Department of Energy. This research is supported by the ÚNKP-19-3 New National Excellence Program of the Ministry for Innovation and Technology, the National Research, Development and Innovation Office of Hungary (K 124845, K 128713, and K 128786) and the Hungarian Academy of Sciences "Lendület" (Momentum) Program (LP 2015-7/2015).

Conflicts of Interest: The author declares no conflict of interest. The funders had no role in the design of the study; in the collection, analyses, or interpretation of data; in the writing of the manuscript, or in the decision to publish the results.

\section{References}

1. Cugnon, J.; Volant, C.; Vuillier, S. Improved intranuclear cascade model for nucleon-nucleus interactions. Nucl. Phys. A 1997, 620, 475. [CrossRef]

2. Ferrari, A.; Sala, P.R.; Ranft, J.; Roesler, S. Cascade particles, nuclear evaporation, and residual nuclei in high-energy hadron-nucleus interactions. Z. Phys. C Part. Fields 1996, C70, 413. [CrossRef]

3. Berman, B.L.; Fultz, S.C. Measurements of the giant dipole resonance with monoenergetic photons. Rev. Mod. Phys. 1975, 47, 713. [CrossRef] 
4. Sikler, F. Centrality Control of Hadron Nucleus Interactions by Detection of Slow Nucleons. Available online: https:/ / arxiv.org/abs/hep-ph/0304065 (accessed on 5 October 2019).

5. Adler, C.; et al. [STAR Collaboration]. Coherent $\rho^{0}$ production in ultraperipheral heavy ion collisions. Phys. Rev. Lett. 2002, 89, 272302. [CrossRef] [PubMed]

6. Adams, J.; et al. [STAR Collaboration]. Production of $\mathrm{e}^{+} \mathrm{e}^{-}$pairs accompanied by nuclear dissociation in ultra-peripheral heavy ion collision. Phys. Rev. C 2004, 70, 031902. [CrossRef]

7. Abelev, B.; et al. [STAR Collaboration]. Centrality determination of $\mathrm{Pb}-\mathrm{Pb}$ collisions at $\sqrt{s_{N N}}=2.76 \mathrm{TeV}$ with ALICE. Phys. Rev. C 2013, 88, 044909. [CrossRef]

8. Adam, J.; et al. [ALICE Collaboration]. Centrality dependence of particle production in $\mathrm{p}-\mathrm{Pb}$ collisions at $\sqrt{s_{\mathrm{NN}}}=5.02 \mathrm{TeV}$. Phys. Rev. C 2015, 91, 064905. [CrossRef]

9. Adriani, O.; Berti, E.; Bonechi, L.; Bongi, M.; Castellini, G.; D'Alessandro, R.; Del Prete, M.; Haguenauer, M.; Itow, Y.; Kasahara, K.; et al. Measurement of very forward neutron energy spectra for $7 \mathrm{TeV}$ proton-proton collisions at the Large Hadron Collider. Phys. Lett. B 2015, 750, 360. [CrossRef]

10. Ueno, M. Zero degree neutron energy spectra measured by the LHCf at $\sqrt{s}=13 \mathrm{TeV}$ proton-proton collision. PoS 2018, ICRC2017, 322.

11. Bass, S.A.; Belkacem, M.; Bleicher, M.; Brandstetter, M.; Bravina, L.; Ernst, C.; Gerland, L.; Hofmann, M.; Hofmann, S.; Konopka, J.; et al. Microscopic models for ultrarelativistic heavy ion collisions. Prog. Part. Nucl. Phys. 1998, 41, 255. [CrossRef]

12. Galoyan, A.; Ribon, A.; Uzhinsky, V. Simulation of neutron production in hadron-nucleus and nucleus-nucleus interactions in Geant4. In Proceedings of the 24th International Baldin Seminar on High Energy Physics Problems: Relativistic Nuclear Physics and Quantum Chromodynamics (ISHEPP 2018) Dubna, Russia, 17-22 September 2018.

13. Allison, J.; Amakoca, K.; Apostolakisd, J.; Arcee, P.; Asaif, M.; Asog, T.; Baglih, E.; Bagulyai, A.; Banerjeej, S.; Barrand, G.; et al. Recent developments in Geant4. Nucl. Instrum. Meth. A 2016, 835, 186. [CrossRef]

14. Weil, J.; Steinberg, V.; Staudenmaier, J.; Pang, L.G.; Oliinychenko, D.; Mohs, J.; Kretz, M.; Kehrenberg, T.; Goldschmidt, A.; Bäuchle, B.; et al. Particle production and equilibrium properties within a new hadron transport approach for heavy-ion collisions. Phys. Rev. 2016, C94, 054905. [CrossRef]

15. Nara, Y.; Otuka, N.; Ohnishi, A.; Niita, K.; Chiba, S. Study of relativistic nuclear collisions at AGS energies from $\mathrm{p}+\mathrm{Be}$ to $\mathrm{Au}+\mathrm{Au}$ with hadronic cascade model. Phys. Rev. C 2000, 61, 024901. [CrossRef]

16. Cassing, W.; Bratkovskaya, E.L. Parton transport and hadronization from the dynamical quasiparticle point of view. Phys. Rev. 2008, C78, 034919. [CrossRef]

17. Grachov, O.A.; Murray, M.J.; Ayan, A.S.; Debbins, P.; Norbeck, E.; Onel, Y.; d'Enterria, D.G. Status of zero degree calorimeter for CMS experiment. AIP Conf. Proc. 2006, 867, 258.

18. Grachov, O.A.; Grachov, O.A.; Metzler, B.; Murray, M.; Snyder, J.; Stiles, L.; Wood, J.; Zhukova, V.; Beaumont, W.; Ochesanu, S.; et al. Measuring photons and neutrons at zero degrees in CMS. Int. J. Mod. Phys. E 2007, 16, 2137. [CrossRef]

19. Grachov, O.A.; Murray, M.; Snyder, S.; Wood, J.; Zhukova, V.; Ayan, A.S.; Debbins, P.; Ingram,D.F.; Norbeck, E.; Onel, Y.; et al. Performance of the combined zero degree calorimeter for CMS. J. Phys. Conf. Ser. 2009, 160, 012059. [CrossRef]

20. Grachov, O.; Murray, M.; Wood, J.; Onel, Y.; Sen, S.; Yetkin, T. Commissioning of the CMS zero degree calorimeter using LHC beam. J. Phys. Conf. Ser. 2011, 293, 012040. [CrossRef]

21. CMS Collaboration. The Performance of CMS ZDC Detector in 2016; CMS Detector Performance Summary; CMS-DP-2018-025; CMS Collaboration: 2017. Available online: https:/ / cds.cern.ch/record/2621978?ln=en (accessed on 9 October 2019).

22. Jowett, J.M. The LHC as a Nucleus-Nucleus Collider. J. Phys. G 2008, 35, 104028. [CrossRef]

23. Tikhonov, A.N. Solution of incorrectly formulated problems and the regularization method. Soviet Math. 1963, 4, 1035.

(c) 2019 by the authors. Licensee MDPI, Basel, Switzerland. This article is an open access article distributed under the terms and conditions of the Creative Commons Attribution (CC BY) license (http:/ / creativecommons.org/licenses/by/4.0/). 